\title{
Diffractive Deeply Inelastic Scattering of Hadronic States with Small Transverse Size
}

\author{
F. Hautmann, ${ }^{1}$ Z. Kunszt, ${ }^{2}$ and D. E. Soper ${ }^{1}$ \\ ${ }^{1}$ Institute of Theoretical Science, University of Oregon, Eugene, Oregon 97403 \\ ${ }^{2}$ Institute of Theoretical Physics, ETH, CH-8093 Zürich, Switzerland
}

(Received 8 June 1998)

\begin{abstract}
Diffractive deeply inelastic scattering from a hadron is described in terms of diffractive quark and gluon distributions. If the transverse size of the hadronic state is sufficiently small, these distributions are calculable using perturbation theory. We present such a calculation and discuss the underlying dynamics. We comment on the relation between this dynamics and the pattern of scaling violation observed in the hard diffraction of large-size states at the DESY ep collider HERA. [S0031-9007(98)07390-6]
\end{abstract}

PACS numbers: $13.60 . \mathrm{Hb}, 12.38 . \mathrm{Bx}$

The data [1,2] from the DESY ep collider HERA on diffractive deeply inelastic scattering can be interpreted $[2,3]$ in terms of diffractive parton distribution functions,

$$
\frac{d f_{a / A}^{\mathrm{diff}}\left(\xi, x_{\mathbb{P}}, t, \mu\right)}{d x_{\mathbb{P}} d t} .
$$

This function gives the joint probability per unit $d \xi$ to find a parton of type $a$ in a hadron of type $A$ and to find that the hadron is diffractively scattered. Specifically, the hadron appears in the final state having lost a fraction $x_{\mathbb{P}}$ of its longitudinal momentum, with $t$ being the invariant momentum transfer. The parton, measured at a scale $\mu$, carries a fraction $\xi$ of the longitudinal momentum of the proton, or a fraction $\beta=\xi / x_{\mathbb{P}}$ of the total longitudinal momentum transferred from the hadron. Diffractive parton distribution functions [4] are also called "extended fracture functions" in the recent paper [5]. These are closely related to the "fracture functions" introduced earlier in [6].

The experimental results for the diffractive deeply inelastic structure function $F_{2}^{\text {diff }}$ are related to the diffractive parton distributions by $[4,7]$

$$
\begin{aligned}
F_{2}^{\mathrm{diff}}\left(x, Q^{2}, x_{\mathbb{P}}, t\right)= & \sum_{a} \int_{a} d \xi \frac{d f_{a / A}^{\mathrm{diff}}\left(\xi, x_{\mathbb{P}}, t, \mu\right)}{d x_{\mathbb{P}} d t} \\
& \times \hat{F}_{a}\left(x / \xi, Q^{2} / \mu^{2}\right) .
\end{aligned}
$$

Here the hard scattering function $\hat{F}_{a}$ is the same as in ordinary deeply inelastic scattering. Note that the contribution from the diffractive gluon distribution can be significant in this formula. Although $\hat{F}_{g}$ is of order $\alpha_{s}$, while $\hat{F}_{q}$ begins at order $\alpha_{s}^{0}$, one may expect that diffractive scattering is related to gluon exchange, so that $f_{g / A}^{\text {diff }} \gg f_{q / A}^{\text {diff }}$. Note also that the diffractive parton distribution functions depend on a factorization scale $\mu$ (which is typically set equal to $Q$ ). The $\mu$ dependence of $f^{\text {diff }}$ follows from the $\mu$ dependence of $\hat{F}$. That is, the diffractive parton distributions obey the ordinary perturbative DGLAP evolution equation. Since $\hat{F}$ is known and the evolution is known, the diffractive parton distributions at a starting scale $\mu_{0}$ can be determined from the experimental results $[2,3]$.

0031-9007/98/81(16)/3333(4)\$15.00
The diffractive parton distributions at $\mu_{0}$ are not perturbatively calculable. Nevertheless, one would like to have some theoretically based insight into their behavior. To this end, notice that the problem lies with the large transverse size of the proton. The diffractive parton distributions for a physically small state would, in principle, be perturbatively calculable. Quarkonium, for instance, would do. Let us consider a slightly simpler model. Replace the proton by a special photon that couples only to a heavy quark of mass $M \gg 1 \mathrm{GeV}$ with a $\gamma^{\mu}$ coupling. Then the diffractive gluon distribution can be represented in terms of cut Feynman diagrams such as the diagram shown in Fig. 1. The top part of this diagram represents the operator that defines the modified minimal subtraction ( $\overline{\mathrm{MS}}$ ) gluon distribution, as specified in more detail below. The lower part represents the coupling of the photon to the heavy $Q \bar{Q}$ state, which couples again to the diffractively scattered photon in the final state. This is but one of many relevant diagrams.

In this paper, we investigate this model for the diffractive gluon and quark distributions, taking the limit $x_{\mathbb{P}} \rightarrow$ 0 in which "pomeron exchange" dominates. Diagrams of order $\alpha_{s}^{4}$ (as in Fig. 1) are the lowest order diagrams that make leading contributions, proportional to $\left(1 / x_{\mathbb{P}}\right)^{2}$ as $x_{\mathbb{P}} \rightarrow 0$. We find that we can take the limit $x_{\mathbb{P}} \rightarrow 0$ inside the integrals that represent the Feynman diagrams, and express the sum of the contributing diagrams as a simple integral that we can evaluate by numerical integration.

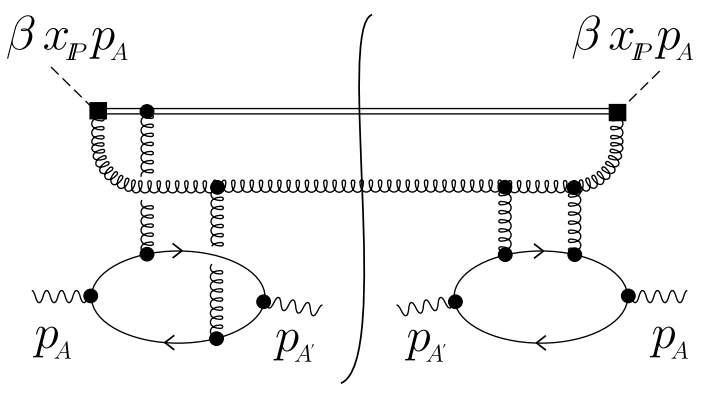

FIG. 1. A typical Feynman graph contributing to the diffractive deeply inelastic scattering of the model vector meson.

(C) 1998 The American Physical Society

3333 
We thus obtain an answer for the diffractive gluon and quark distribution functions at a starting scale $\mu_{0} \approx M$.

Evidently, the heavy quark state in the model vector meson is not the same as the light quark state in a proton. Nevertheless, since the model is based on non-Abelian gauge theory, the qualitative features of the resulting diffractive parton distributions may give us hints about the real world. After giving the formulas that express the solution of the model, we explore these qualitative features.

Consider diffractive deeply inelastic scattering of a hadron $A$. Let $\left(p_{A}, s_{A}\right)$ and $\left(p_{A^{\prime}}, s_{A^{\prime}}\right)$ denote the momentum and spin of the incident and the diffracted hadron. Let us work in a frame in which the incident hadron is boosted along the positive light-cone direction and let $p_{A}^{+}$ be its "plus" momentum. The four-momentum transfer $q \equiv p_{A}-p_{A^{\prime}}$ has components $q^{\mu}=\left(q^{+}, q^{-}, \mathbf{q}\right)$. (Note that in our notation $q$ is not the virtual photon momentum, as is usual in the literature.) The diffracted hadron can be characterized by the fractional loss of longitudinal momentum $x_{\mathbb{P}}=q^{+} / p_{A}^{+}$and the invariant momentum transfer $t=\left(p_{A}-p_{A^{\prime}}\right)^{2}$. For small $x_{\mathbb{P}}, t$ is approximately given by $t \simeq-\mathbf{q}^{2}$.

Consider the definition of the diffractive parton distributions in terms of matrix elements of bilocal field operators [4]. This is the same definition [8] as for inclusive parton distributions except that one requires that the final state include the diffractively scattered hadron. For gluons one has

$$
\begin{aligned}
\frac{d f_{g / A}^{\mathrm{diff}}\left(\xi, x_{\mathbb{P}}, t, \mu\right)}{d x_{\mathbb{P}} d t}= & \frac{1}{16 \pi^{2}} \frac{1}{2 \pi \xi p_{A}^{+}} \frac{1}{2} \sum_{s_{A}} \int d y^{-} e^{-i \xi p_{A}^{+} y^{-}} \sum_{X, s_{A^{\prime}}}\left\langle p_{A}, s_{A}\left|\widetilde{F}_{a}\left(0, y^{-}, \mathbf{0}\right)^{+\nu}\right| p_{A^{\prime}}, s_{A^{\prime}} ; X\right\rangle \\
& \times\left\langle p_{A^{\prime}}, s_{A^{\prime}} ; X\left|\widetilde{F}_{a}(0)_{\nu}{ }^{+}\right| p_{A}, s_{A}\right\rangle,
\end{aligned}
$$

where $\tilde{F}_{a}\left(0, y^{-}, \mathbf{0}\right)^{+\nu}$ is the field strength operator modified by multiplication by an exponential of a line integral of the vector potential:

$$
\widetilde{F}_{a}\left(0, y^{-}, \mathbf{0}\right)^{\mu \nu}=\left[\mathcal{P} \exp \left(i g \int_{y-}^{\infty} d x^{-} A_{c}^{+}\left(0, x^{-}, \mathbf{0}\right) t_{c}\right)\right]_{a b} F_{b}\left(0, y^{-}, \mathbf{0}\right)^{\mu \nu} .
$$

The symbol $\mathcal{P}$ denotes path ordering of the exponential. The matrices $t_{c}$ in Eq. (4) are the generators of the adjoint representation of SU(3). The operator product in Eq. (3) has ultraviolet divergences. It is understood that these are renormalized at the scale $\mu$ using the $\overline{\mathrm{MS}}$ prescription.

Similarly, for quarks of type $j$ one has

$$
\begin{aligned}
\frac{d f_{j / A}^{\mathrm{diff}}\left(\xi, x_{\mathbb{P}}, t, \mu\right)}{d x_{\mathbb{P}} d t}= & \frac{1}{16 \pi^{2}} \frac{1}{4 \pi} \frac{1}{2} \sum_{s_{A}} \int d y^{-} e^{-i \xi p_{A}^{+} y^{-}} \sum_{X, s_{A^{\prime}}}\left\langle p_{A}, s_{A}\left|\tilde{\bar{q}}_{j}\left(0, y^{-}, \mathbf{0}\right)\right| p_{A^{\prime}}, s_{A^{\prime}} ; X\right\rangle \gamma^{+} \\
& \times\left\langle p_{A^{\prime}}, s_{A^{\prime}} ; X\left|\tilde{q}_{j}(0)\right| p_{A}, s_{A}\right\rangle,
\end{aligned}
$$

where $\tilde{q}_{j}\left(0, y^{-}, \mathbf{0}\right)$ is given by

$$
\begin{aligned}
\tilde{q}_{j}\left(0, y^{-}, \mathbf{0}\right)= & {\left[\mathcal{P} \exp \left(i g \int_{y-}^{\infty} d x^{-} A_{c}^{+}\left(0, x^{-}, \mathbf{0}\right) t_{c}\right)\right] } \\
& \times q_{j}\left(0, y^{-}, \mathbf{0}\right),
\end{aligned}
$$

with $t_{c}$ being the generators of the fundamental representation of SU(3).

Consider now the case in which $A$ is a photon coupling to heavy quarks, as discussed above. Let us consider small longitudinal momentum transfers, $x_{\mathbb{P}} \ll 1$, and let us examine leading-power contributions in $x_{\mathbb{P}}$. These contributions first appear in perturbation theory in graphs of the same order as the one shown in Fig. 1. We study the full set of graphs at this order. To perform this study, we use the light cone gauge $A^{-}=0$. A discussion of the gauge choice and the details of our analysis will be given elsewhere [9]. Here we limit ourselves to reporting the main results and discussing their implications. To the leading power in $x_{\mathbb{P}}$ we find that the matrix elements (3) and (5) can be written in the following form $(a=q, g)$ :

$$
\begin{aligned}
\frac{d f_{a / A}^{\mathrm{diff}}\left(\beta, x_{\mathbb{P}}, \mathbf{q}^{2}, M\right)}{d x_{\mathbb{P}} d t}= & \frac{1}{64 \pi^{2}} \frac{1}{2} \sum_{\varepsilon} \sum_{\varepsilon^{\prime}} \int \frac{d^{2} \mathbf{s}}{(2 \pi)^{2}} \frac{1}{\mathbf{s}^{2}(\mathbf{q}+\mathbf{s})^{2}} \int \frac{d^{2} \mathbf{s}^{\prime}}{(2 \pi)^{2}} \frac{1}{\mathbf{s}^{\prime 2}\left(\mathbf{q}+\mathbf{s}^{\prime}\right)^{2}} L\left(\mathbf{q}, \mathbf{s}, M, \varepsilon, \varepsilon^{\prime}\right) \\
& \times U_{a}\left(x_{\mathbb{P}}, \beta, \mathbf{q}, \mathbf{s}, \mathbf{s}^{\prime}\right) L\left(\mathbf{q}, \mathbf{s}^{\prime}, M, \varepsilon, \varepsilon^{\prime}\right) .
\end{aligned}
$$

This result can be interpreted in terms of graphs with exchange of two gluons in a color-singlet state (such as the one depicted in Fig. 1). In $A^{-}=0$ gauge, only such graphs contribute leading power terms as $x_{\mathbb{P}} \rightarrow 0$. The denominators in the first line of Eq. (7) come from the gluon propagators. The factor $U$ comes from the colorsinglet projection of the two-gluon irreducible amplitude associated with the insertion of the operators in Eqs. (3) and (5). The factors $L$ describe the coupling of the two gluons to the incoming quark-antiquark system. 
More precisely, the functions $U_{a}$ have the form

$$
\begin{aligned}
U_{a}\left(x_{\mathbb{P}}, \beta, \mathbf{q}, \mathbf{s}, \mathbf{s}^{\prime}\right)= & \frac{g_{s}^{4} c_{a}}{4 \pi \beta(1-\beta) x_{\mathbb{P}}^{2}} \int \frac{d^{2} \mathbf{k}}{(2 \pi)^{2}} \\
& \times \operatorname{Tr}\left[u_{a}^{\dagger}\left(\beta, \mathbf{k}, \mathbf{q}, \mathbf{s}^{\prime}\right) u_{a}(\beta, \mathbf{k}, \mathbf{q}, \mathbf{s})\right] .
\end{aligned}
$$

Here $c_{a}$ is the color factor and is given for quarks and gluons by

$$
c_{q}=C_{F}^{2} N_{c}, \quad c_{g}=C_{A}^{2}\left(N_{c}^{2}-1\right) .
$$

The function $u_{a}$ can be written as

$$
\begin{aligned}
u_{a}= & \psi_{a}(\mathbf{k}, \mathbf{k})-\psi_{a}(\mathbf{k}, \mathbf{k}+\mathbf{s}) \\
& +\psi_{a}(\mathbf{k}, \mathbf{k}-\mathbf{q})-\psi_{a}(\mathbf{k}, \mathbf{k}-\mathbf{q}-\mathbf{s}) .
\end{aligned}
$$

For $a=q$, the function $\psi$ has the following expression in terms of the $2 \times 2$ Pauli $\sigma$ matrices:

$$
\psi_{q}(\mathbf{k}, \mathbf{p})=\frac{\sqrt{\beta(1-\beta) \mathbf{k}^{2}}}{\beta \mathbf{k}^{2}+(1-\beta) \mathbf{p}^{2}} \mathbf{p} \cdot \sigma .
$$

For $a=g$, the function $\psi$ is expressed in terms of two transverse vector indices $i, j=1,2$ as

$$
\psi_{g}^{i j}(\mathbf{k}, \mathbf{p})=\frac{\beta \mathbf{k}^{2} \delta^{i j}+2(1-\beta) \mathbf{p}^{i} \mathbf{p}^{j}}{\beta \mathbf{k}^{2}+(1-\beta) \mathbf{p}^{2}}
$$

The functions $L$ in Eq. (7) are given by

$$
\begin{aligned}
L\left(\mathbf{q}, \mathbf{s}, M, \varepsilon, \varepsilon^{\prime}\right)=\frac{e_{Q}^{2} e^{2} g_{s}^{2}}{4 \pi} \int \frac{d^{2} \mathbf{r}}{(2 \pi)^{2}} \int_{0}^{1} d z \operatorname{Tr}\{ & {\left[-\Phi^{\dagger}\left(z, \mathbf{r}+\mathbf{s}+z \mathbf{q}, M, \varepsilon^{\prime}\right)-\Phi^{\dagger}\left(z, \mathbf{r}-\mathbf{s}-(1-z) \mathbf{q}, M, \varepsilon^{\prime}\right)\right.} \\
& \left.\left.+\Phi^{\dagger}\left(z, \mathbf{r}+z \mathbf{q}, M, \varepsilon^{\prime}\right)+\Phi^{\dagger}\left(z, \mathbf{r}-(1-z) \mathbf{q}, M, \varepsilon^{\prime}\right)\right] \Phi(z, \mathbf{r}, M, \varepsilon)\right\},
\end{aligned}
$$

where $e_{Q}$ is the quark electric charge in units of $e=$ $\sqrt{4 \pi \alpha}$ and

$$
\begin{aligned}
\Phi(z, \mathbf{k}, M, \varepsilon)=\frac{1}{\left(\mathbf{k}^{2}+M^{2}\right)}[ & (1-z) \varepsilon \cdot \sigma \mathbf{k} \cdot \sigma \\
& -z \mathbf{k} \cdot \sigma \varepsilon \cdot \sigma+i M \varepsilon \cdot \sigma],
\end{aligned}
$$

with $\varepsilon$ and $\varepsilon^{\prime}$ being the initial and final photon transverse polarizations.

In Eq. (7), the Green's functions $U_{a}$ are universal: as long as the diffracted system is small enough that lowest order perturbation theory applies, these functions control the scattering. The dependence on the specific diffracted system is contained in the functions $L$. In Ref. [9], we will utilize a selection of hadronic functions $L$.

The explicit form of the Green's function $U_{a}$ is given in Eqs. (8)-(12). In the framework of $x^{-}$-ordered perturbation theory, the function $\psi$ in Eq. (10) can be interpreted as the wave function for an effective quark or gluon state created by the operator in Eqs. (3) or (5). (Here the line integral of $A^{+}$in Eqs. (4) and (6) represents the interaction of an effective infinite-momentum parton with the gluon field.) The wave function description is natural in approaches that look at diffractive scattering in a frame in which this quark or gluon system has large "minus" momentum [9-12]. Note that each of the terms in Eq. (10) would give rise to an ultravioletdivergent integration over $\mathbf{k}$ in Eq. (8), but that the bad behavior cancels among the terms. This is because $\mathbf{k}^{2} \rightarrow$ $\infty$ corresponds to the partons being at the same transverse position. But since the net color of the state is zero, the coupling of the state to gluons vanishes in this limit.

At the leading power level, the $x_{\mathbb{P}}$ dependence of the diffractive parton distributions is given simply by the overall factor $1 / x_{\mathbb{P}}^{2}$ that we have factored from the integral in Eq. (8). The $\beta$ and $t$ (or $\mathbf{q}^{2}$ ) dependences, on the other hand, are nontrivial and come from the factors in the integrand in the right-hand side of Eq. (7).
The only explicit $\beta$ dependence in this integrand, in particular, is contained in the functions $U_{a}$. Inspection of Eqs. (8) $-(12)$ shows that, for $\beta \rightarrow 0$, the quark and gluon Green's functions behave, respectively, as $U_{q} \sim \operatorname{const} \beta^{0}$ and $U_{g} \sim$ const $\beta^{-1}$. For $\beta \rightarrow 1$, the functions $U_{a}$ have a constant behavior $(1-\beta)^{0}$ at finite $\mathbf{q}^{2}, \mathbf{s}^{2}$, and $\mathbf{s}^{\prime 2}$.

We now evaluate the diffractive distributions by performing numerically the integrations (7), (8), (13). In Fig. 2 we report the results by plotting the $\beta$ dependence

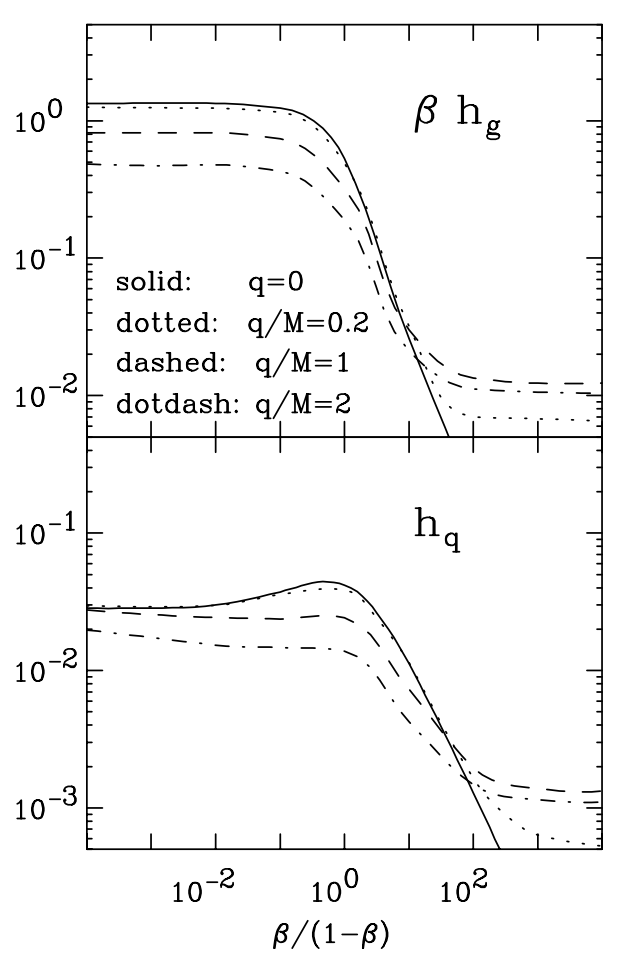

FIG. 2. The $\beta$ dependence of the gluon (above) and quark (below) diffractive distributions for different values of $\mathbf{q}^{2} \simeq|t|$. The rescaled distributions $h_{a}$ are defined as $h_{a}\left(\beta, \mathbf{q}^{2} / M^{2}\right)=x_{\mathbb{P}}^{2} M^{2}\left[d f_{a / A}^{\mathrm{diff}} /\left(d x_{\mathbb{P}} d t\right)\right] /\left(\alpha^{2} e_{Q}^{4} \alpha_{s}^{4}\right)$. 


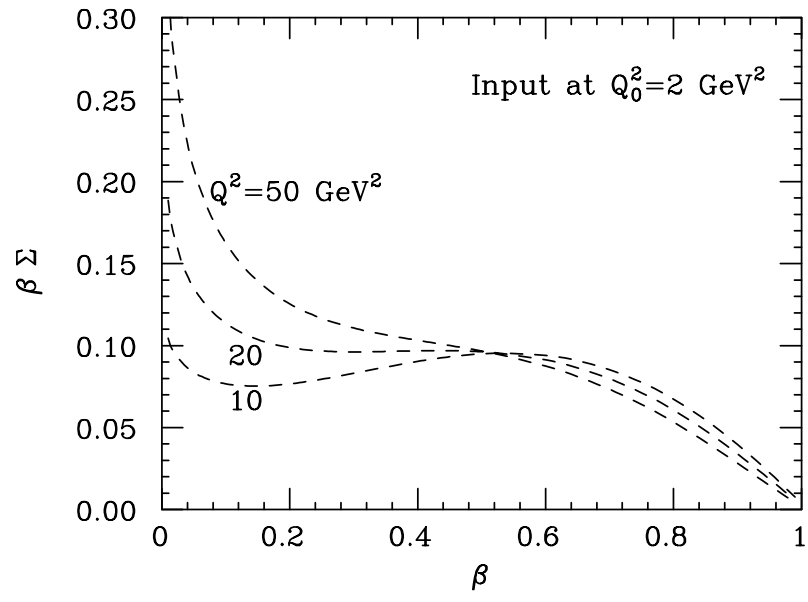

FIG. 3. $Q^{2}$ evolution of the diffractive singlet quark distribution $\Sigma\left(\beta, Q^{2}\right)=\mathcal{N} \sum_{j} \int_{0}^{M^{2}} d \mathbf{q}^{2} d f_{j / A}^{\text {diff }} /\left(d x_{\mathbb{P}} d t\right)$, where $\mathcal{N}=$ $x_{\mathbb{P}}^{2} /\left(\alpha^{2} e_{Q}^{4} \alpha_{s}^{4}\right)$ and the sum runs over $j=\{u, \bar{u}, d, \bar{d}, \ldots\}$.

of the quark and gluon diffractive distributions for different values of $\mathbf{q}$. To emphasize the regions of small $\beta$ and large $\beta$ we make a logarithmic plot in the variable $\beta /(1-\beta)$. The curves in Fig. 2 reflect the behavior of the functions $U_{a}$ discussed above. In particular, as $\beta \rightarrow 1$ the $\mathbf{q} \neq 0$ distributions have a constant behavior. The asymptotic constants, on the other hand, are small compared to the values of the distributions at intermediate $\beta$. Correspondingly, the diffractive distributions fall off as one approaches the small $(1-\beta)$ region. Note that the gluon distribution is much larger than the quark distribution. Roughly, the different order of magnitude is accounted for by the color factors in Eq. (9), $c_{g} / c_{q}=27 / 2$.

The calculation described so far does not have scaling violation. One may interpret the results above as a model for the diffractive distribution functions at a scale of order $M^{2}$ and study the scale dependence due to renormalization group evolution. In Fig. 3 we report the result of using the ordinary evolution equations for the evolution of the initial distributions up to different values of $Q^{2}$. In this figure we plot the flavor-singlet quark distribution. In leading order this is proportional to the structure function $F_{2}^{\text {diff }}$. Here we have assumed the initial scale and the mass to be $Q_{0}^{2}=M^{2}=2 \mathrm{GeV}^{2}$ (about the charm quark mass squared) and we have integrated the distributions $d f_{a / A}^{\text {diff }} /\left[d x_{\mathbb{P}} d t\right]$ over $t \simeq-\mathbf{q}^{2}$ from 0 to $M^{2}$.

Is there any relation between these calculations and the data from diffractive deeply inelastic scattering at HERA? Obviously, the protons probed in experiments at HERA have a large transverse size, in contrast to the small-size hadronic state considered in our calculation. Nevertheless, we find, by studying different hadronic wave functions [9], that the $\beta$ behavior as well as the relative size of the singlet quark and gluon distributions depends only weakly on the specific nature of the incoming hadronic state. This suggests that qualitative features of the results for small-size hadrons may apply with more generality.
In particular, one of the most peculiar features of the HERA data [1,2] is the striking difference in the $Q^{2}$ evolution between the diffractive and the inclusive structure functions. In our calculation, the diffractive quark distribution grows with $Q^{2}$ at low $\beta$ and decreases at high $\beta$ (Fig. 3). The stability point at which the behavior changes is $\beta \approx 0.5$. This is in striking contrast with the case of the inclusive quark distribution in a proton, for which the stability point is at $x \approx 0.08$, but is in qualitative agreement with the behavior found in the HERA experiments. The explanation for growth in the quark distribution up to such large values of $\beta$ in the diffractive case is that the initial gluon distribution (Fig. 2) is large even at large values of $\beta$. As $Q^{2}$ increases, the gluons feed the quark distribution through the splitting $g \rightarrow q \bar{q}$.

This research is supported in part by the U.S. Department of Energy Grant No. DE-FG03-96ER40969. Z. K. acknowledges the hospitality of the Institute of Theoretical Science at the University of Oregon and the Theory Division at CERN while part of this work was being done.

[1] ZEUS Collaboration, M. Derrick et al., Phys. Lett. B 315, 481 (1993); 332, 228 (1994); 338, 483 (1994); Z. Phys. C 68, 569 (1995); H1 Collaboration, T. Ahmed et al., Nucl. Phys. B429, 477 (1994); Phys. Lett. B 348, 681 (1995).

[2] H1 Collaboration, C. Adloff et al., Z. Phys. C 76, 613 (1997); ZEUS Collaboration, M. Derrick et al., Phys. Lett. B 356, 129 (1995); Z. Phys. C 68, 569 (1995); 70, 391 (1996); J. Breitweg et al., Eur. Phys. J. C1, 81 (1998).

[3] T. Gehrmann and W. J. Stirling, Z. Phys. C 70, 89 (1996); K. Golec-Biernat, and J. Kwiecinski, Phys. Lett. B 353, 329 (1995); Z. Kunszt and W. J. Stirling, in Proceedings of the International Workshop on Deep Inelastic Scattering DIS96, Rome, Italy, 1996, edited by G. D'Agostini, and A. Nigro (World Scientific, Singapore, 1997), p. 240; L. Alvero, J.C. Collins, J. Terron, and J. Whitmore, hep-ph/9701374; hep-ph/9805268; D. de Florian and R. Sassot, Phys. Rev. D 58, 54003 (1998).

[4] A. Berera and D. E. Soper, Phys. Rev. D 53, 6162 (1996).

[5] M. Grazzini, L. Trentadue, and G. Veneziano, Nucl. Phys. B519, 394 (1998).

[6] L. Trentadue and G. Veneziano, Phys. Lett. B 323, 201 (1994).

[7] J. C. Collins, Phys. Rev. D 57, 3051 (1998).

[8] J.C. Collins and D.E. Soper, Nucl. Phys. B194, 445 (1982); G. Curci, W. Furmanski, and R. Petronzio, Nucl. Phys. B175, 27 (1980).

[9] F. Hautmann, Z. Kunszt, and D. E. Soper (to be published).

[10] J. D. Bjorken and J. Kogut, Phys. Rev. D 8, 1341 (1973); L. Frankfurt and M. Strikman, Phys. Rep. 160, 235 (1988).

[11] N. N. Nikolaev and B. G. Zakharov, Z. Phys. C 49, 607 (1991); 53, 331 (1992); M. Wüsthoff, Phys. Rev. D 56, 4311 (1997).

[12] W. Buchmüller and A. Hebecker, Nucl. Phys. B476, 203 (1996); A. Hebecker, Nucl. Phys. B505, 349 (1997). 